\title{
PRESTÍGIO PROFISSIONAL DO ENFERMEIRO: ESTIMAÇÃO DE MAGNITUDES E DE CATEGORIAS EXPANDIDAS
}

Fátima Aparecida Emm Faleiros Sousa ${ }^{1}$ José Aparecido da Silva ${ }^{2}$

Sousa FAEF, Silva JA. Prestígio profissional do enfermeiro: estimação de magnitudes e de categorias expandidas. Rev Latino-am Enfermagem 2001 novembro-dezembro; 9(6):19-24.

O prestígio social de profissões de nível superior exercidas por: assistente social, biólogo, dentista, enfermeiro, engenheiro, farmacêutico, físico, fisioterapeuta, fonoaudiólogo, médico, psicólogo, químico e sociólogo foi escalonado pelos métodos psicofísicos de estimação de magnitude e de categorias expandidas. Os resultados mostraram que: 1) quando aumentamos a amplitude limitada das categorias, esse método passa a ter as mesmas características da estimação de magnitudes 2) a relação entre as estimativas de magnitudes e as estimativas de categorias expandidas é uma função de potência com um expoente não significativamente diferente de 1,0. Em função desses dados, podemos concluir que: (1) a profissão de Enfermeiro ocupa a sétima ou a oitava posição em termos do prestígio das 13 profissões, enquanto a de médico ocupa a primeira posição nas escalas obtidas pelos métodos utilizados; (2) as ordenações resultantes dos métodos produzem posições de prestígio altamente concordantes para as diferentes profissões.

DESCRITORES: psicofísica, mensuração, pesquisa

\section{NURSES' PROFESSIONAL PRESTIGE: ESTIMATION OF MAGNITUDES AND EXPANDED CATEGORIES}

The prestige of professionals such as social workers, biologists, dentists, nurses, engineers, pharmacists, physicists, physical therapists, speech-language pathologists, physicians, psychologists, chemists and sociologists was scaled by the psychophysical methods of estimation of magnitudes and expanded categories. Results showed that: 1) when we increase the limited amplitude of categories, this method has the same characteristics as those of the estimation of magnitudes. 2) the relationship between the estimations of magnitudes and estimations of expanded categories is a power function with an exponent that is not significantly different from 1.0. These data enabled the following conclusions: 1- The nursing profession is in the seventh or eighth position regarding the prestige of the 13 professions whereas physicians are in the first position in the scale obtained by the used methods; 2- the orders resulting from the methods produce positions of prestige that highly agree for the different professions.

KEY WORDS: psychophysics, measurement, research

\section{PRESTÍGIO PROFESIONAL DEL ENFERMERO: ESTIMACIÓN DE MAGNITUDES Y CATEGORÍAS EXPANDIDAS}

El prestigio social de profesiones de nivel superior ejercidas por: asistentes sociales, biólogos, dentistas, enfermeros, ingenieros, farmacéuticos, físicos, fisioterapeutas, fonoaudiólogos, médicos, psicólogos, químicos y sociólogos fue escalonado por el método psicofísico de estimación de magnitudes y de categorías expandidas. Los resultados mostraron que: 1) cuando aumentamos la amplitud limitada de las categorías, ese método pasa a tener las mismas características de la estimación de magnitudes. 2) la relación entre las estimativas de magnitud y las estimativas de categorías expandidas es una función de potencia con exponente no significativamente diferente de 1.0 En función de estos datos, podemos concluir que: 1- la profesión de enfermero ocupa la séptima o la octava posición en términos de prestigio de las 13 profesiones, mientras que la de médico ocupa la primera posición en las escalas obtenidas por los métodos utilizados; 2- las ordenaciones resultantes de los métodos producen posiciones de prestigio altamente semejantes para las diferentes profesiones.

DESCRIPTORES: psicofísica, medición, investigación

\footnotetext{
${ }^{1}$ Enfermeiro, Professor Associado da Escola de Enfermagem de Ribeirão Preto da Universidade de São Paulo, Centro Colaborador da OMS para o desenvolvimento da pesquisa em enfermagem, e-mail: faleiros@eerp.usp.br; ${ }^{2}$ Psicólogo, Professor Titular da Faculdade de Filosofia, Ciências e Letras de Ribeirão Preto da Universidade de São Paulo
} 


\section{INTRODUÇÃO}

$\boldsymbol{U}_{\mathrm{m}}$ dos primeiros estudos que investigou com métodos psicofísicos escalares o prestígio ocupacional e profissional foi o qual empregou e comparou os métodos de estimação de magnitudes e estimação de categorias para escalonarem uma lista de 100 ocupações $^{(1)}$. Os dados revelaram que a escala de categorias de prestígio ocupacional é uma função aproximadamente logarítmica da escala de estimação de magnitudes, quando os sujeitos não limitaram a amplitude de julgamentos das profissões consideradas de mais alto prestígio. Analisou-se também separadamente a relação existente entre estimativas de categorias e estimativas de magnitudes com ou sem limitação da amplitude de julgamentos das profissões. Esta análise revelou que com amplitude limitada de julgamentos a escala de categorias é uma função linear da escala de magnitudes, indicando que os sujeitos estão fazendo julgamentos de diferenças e não de razões. Com amplitude ilimitada de julgamentos das profissões, a relação entre estas duas escalas é uma função logarítmica, indicando que os sujeitos estão fazendo julgamentos de razões do prestígio profissional. Em adição, os dados mostraram que quando comparadas às escalas de magnitudes de prestígio profissional contendo amplitude limitada dos julgamentos com as escalas de magnitudes contendo amplitude ilimitada, a relação foi curvilínea, indicando que a primeira é uma função logarítmica da segunda, tal como usualmente ocorre nas relações entre estimativas de categorias e estimativas de magnitudes. Em resumo, os dados fortemente indicam que o contínuo de prestígio profissional tem características protéticas $^{(1)}$. Mas, também demonstram que a amplitude é uma variável muito importante nesta indicação, uma vez que quando a mesma é ilimitada, os resultados das estimativas de magnitudes sustentam que os sujeitos estão julgando razões entre os estímulos. 0 contrário é verdadeiro, quando são limitadas, os resultados das estimativas indicam que os sujeitos estão efetuando julgamentos de diferenças entre os estímulos. Em outras palavras, a relação logarítmica entre estimativas de magnitudes e estimativas de categorias somente é obtida quando a amplitude das primeiras é ilimitada.

Em outro estudo, foram usados dois métodos escalares diretos (estimação de magnitudes e estimação de razões) e um método indireto (comparação aos pares) para construírem escalas de preferências ocupacionais ${ }^{(2)}$. Neste estudo participaram 74 estudantes universitários os quais julgaram 17 diferentes ocupações pertencentes, segundo as normas estatísticas suecas, a três diferentes grupos sociais. Os resultados mostraram que as escalas de preferências ocupacionais construídas através dos métodos diretos de estimação de magnitudes e de estimação de razões foram linearmente relacionadas. A escala construída através do método indireto de comparação aos pares foi uma função logarítmica da escala de estimação de magnitudes e também da escala de estimação de razões. Também, similarmente ao que ocorre com contínuos métricos, a variabilidade expressa pelo desvio padrão da média é uma função linear das estimativas de magnitudes, comprovando assim a Lei de Ekman. Em resumo, os dados suportam que o contínuo de preferência ocupacional tem características protéticas ${ }^{(2)}$. Estudo similar foi realizado utilizando uma amostra de 1.796 sujeitos que avaliaram a preferência de 16 ocupações diferentes através dos métodos de estimação de categorias e de estimação de magnitudes ${ }^{(3)}$. Consistente com os dados obtidos com contínuos métricos, foi encontrado que as duas escalas não são linearmente relacionadas entre si, ou seja, o contínuo de preferência ocupacional possui características protéticas.

Foram realizados dois estudos nos quais investigaram através do método de emparelhamento intermodal a preferência ocupacional $^{(4-5)}$. No primeiro estudo, 24 sujeitos emparelharam intensidades de sons e forças dinamométricas a 17 ocupações, as quais foram selecionadas a partir de um outro experimento ${ }^{(2,4)}$. Os emparelhamentos de sons e de forças dinamométricas feitos para as diferentes ocupações foram projetados, em coordenadas logarítmicas, um em função do outro. A inclinação da reta (expoente da função de potência) foi igual a 0,29 , cujo valor foi relativamente próximo daquele de 0,38 predito a partir da razão entre os expoentes característicos para intensidades de sons e forças dinamométricas $(0,64 / 1,70)$, indicando que os sujeitos são capazes de julgar proporcionalmente os estímulos quando esses são emparelhados a sons e a forças dinamométricas. A correlação de ordem de Spearman (rho) entre os emparelhamentos de sons e de forças dinamométricas foi igual a 0,94 , indicando que os sujeitos foram consistentes em seus julgamentos de razões usando duas modalidades diferentes de respostas, uma auditiva e a outra muscular.

No segundo estudo, 38 sujeitos emparelharam duração aparente para indicar a desejabilidade e a indesejabilidade (atributo inverso) das mesmas 17 ocupações utilizadas no estudo anterior ${ }^{(5)}$. Os dados mostraram que a relação entre as médias geométricas dos emparelhamentos de duração aparente de desejabilidade e de indesejabilidade para cada uma das profissões foi uma função de potência com um expoente de $-1,10$, cujo valor é muito próximo daquele esperado de -1,0. Do mesmo modo que os dados médios, os individuais também foram plenamente descritos por funções de potência cujos expoentes foram próximos de $-1,0$, mas variaram entre $-0,49$ a $-2,47$, significando que apesar do expoente médio obtido ser próximo do esperado os expoentes individuais são muito variáveis.

Sob o ponto de vista da teoria geral da mensuração, recentemente analisou-se a maleabilidade das estimativas de razões de prestígio ocupacional ${ }^{(6)}$. Utilizaram 50 sujeitos que fizeram estimativas de razões e de diferenças do prestígio de 12 diferentes ocupações. Seus resultados mostraram que os julgamentos de razões 
e de diferenças foram monotonicamente relacionados e foram consistentes com a hipótese de que os sujeitos usam a mesma operação tanto para julgamentos de diferenças quanto de razões.

Tomado em conjunto, os dados destes experimentos mostram que o contínuo de prestígio e de preferência ocupacionais e profissionais possui características protéticas e que entre estudantes americanos e suecos, a profissão de Médico é a que possui o mais alto prestígio ou é a profissão mais preferida.

Em certo estudo, o prestígio social de profissões de nível superior, exercidas por assistente social, biólogo, dentista, enfermeiro, engenheiro, farmacêutico, físico, fisioterapeuta, fonoaudiólogo, médico, psicólogo, químico e sociólogo foi escalonado pelos métodos psicofísicos de comparação aos pares e estimação de magnitude ${ }^{(7)}$. Os resultados mostraram que os dois métodos produzem escalonamentos substancialmente diferentes; a escala de comparação aos pares (proporção ou z) é uma função logarítmica da escala de estimação de magnitudes; a escala de comparação aos pares (proporção ou z) é uma função linear dos logaritmos das estimativas de magnitudes e uma alta correlação (rho $=0,95$ ) entre os graus de prestígio atribuído às profissões obtidas pelos dois métodos. Em função desses dados, podemos concluir que o contínuo de prestígio social (não métrico) escalonado por estes dois métodos produz uma relação semelhante àquela obtida com contínuos sensoriais (métricos) e o contínuo de prestígio social tem características quantitativas ou protéticas, e não qualitativas ou metatéticas.

Experimento 1: Comparação entre os métodos psicofísicos escalares de estimação de magnitudes e estimação de categorias expandidas.

Neste Experimento, o prestígio profissional do enfermeiro foi avaliado através de dois métodos psicofísicos independentes: estimação de magnitudes e estimação de categorias expandidas. Estes dois métodos psicofísicos (categorias versus magnitudes) diferem num aspecto que pode ser relevante para este problema. No método de estimação de magnitudes, o sujeito seleciona e usa uma amplitude de números que representa sua amplitude subjetiva. Ao contrário, no método de estimação de categorias, o experimentado escolhe arbitrariamente a amplitude de categorias, usualmente menor (embora algumas vezes possa ser maior) do que a amplitude escolhida naturalmente pelo sujeito no método de estimativas de magnitudes (amplitude ilimitada). Se a expressão natural da relação numérica entre as estimativas subjetivas de um número de estímulos depende da amplitude ou do número de categorias disponíveis, o típico método de estimação de categorias (amplitude limitada) pode constranger os julgamentos dos sujeitos. Assim, as estimativas de categorias não representam da mesma maneira que as estimativas de magnitudes, o crescimento das estimativas subjetivas, resultando numa relação não linear entre as duas ${ }^{(8-9)}$

Com estímulos métricos ou aditivos este efeito tem sido realizado ${ }^{(8-10)}$. Os dados destes estudos mostraram nitidamente que o número de categorias afeta a relação entre as estimativas de categorias e as de magnitudes. Este efeito não tem sido ainda verificado com contínuos não métricos. 0 propósito principal deste Experimento foi determinar o efeito da amplitude de categorias disponíveis ao sujeito sobre a função relacionando as estimativas de categorias e as estimativas de magnitudes para o contínuo não métrico de prestígio profissional. Para isso, este contínuo foi escalonado usando dois métodos psicofísicos: os método de estimação de magnitudes e o método de estimação de categorias expandidas (categorias de 1 a 40 derivadas das amplitudes das médias das estimativas de magnitude registradas em um determinado trabalho $\left.{ }^{(7)}\right)$. O ponto principal deste objetivo foi determinar a forma da função relacionada as estimativas de categorias com as estimativas de magnitudes. Os objetivos secundários foram verificar se as ordenações dos graus de prestígios profissionais resultantes dos dois métodos psicofísicos são similares entre si, e verificar se a variabilidade das estimativas de categorias expandidas aumenta linearmente em função do aumento das estimativas de categorias, tal como ocorre com as estimativas de magnitudes.

\section{MÉTODOS}

Participantes

Participaram 45 estudantes universitários dos diferentes cursos de graduação e pós-graduação do Campus da USP de Ribeirão Preto, com idades variando entre 18 e 41 anos. Na tarefa de estimação de magnitudes participaram 23 (18 femininos e 5 masculinos) e na de estimação de categorias expandidas participaram 22 (16 femininos e 6 masculinos) sujeitos. Todos eram ingênuos, ou seja, não conheciam ainda os propósitos do experimento.

Material

Foram elaborados 2 blocos de papel contendo na primeira página instruções específicas para cada tipo de método psicofísico e nas páginas seguintes de 13 profissões (químico, sociólogo, dentista, engenheiro, farmacêutico, biólogo, enfermeiro, médico, físico, assistente social, fisioterapeuta, psicólogo e fonoaudiólogo).

Procedimento

Os métodos escalares utilizados foram estimação de magnitudes e estimação de categorias expandidas. No primeiro 
método, a tarefa do sujeito consistiu em assinalar um número a cada profissão que fosse proporcional à quantidade de prestígio social atribuída às mesmas. Módulo ou estímulo padrão não foram previamente designadas, sendo que cada sujeito estabeleceu apenas 13 estimativas, uma para cada profissão.

No segundo método, a tarefa dos sujeitos foi assinalar uma categoria que variava de 1 a 40, derivada da amplitude das médias das estimativas de magnitudes das diferentes profissões registradas em um determinado estudo ${ }^{(7)}$. Assim, se o sujeito julgasse que uma profissão tem o maior prestígio entre as demais, ele deveria assinalar a ela a categoria máxima de 40 . Se o sujeito julgasse que uma outra profissão tem o menor prestígio, ele deveria assinalar a ela a categoria de mínima de 1. 0 sujeito deveria atribuir as outras categorias intermediárias, ou seja as categorias de 2 a 39 para indicar graus intermediários de prestígio que as outras profissões possuem em nossa sociedade. Previamente não foram indicadas aos sujeitos as profissões de prestígios máximo e mínimo. As treze profissões foram apresentadas uma a uma, em páginas separadas, portanto cada sujeito estabeleceu 13 estimativas, sendo uma para cada profissão. A ordem de distribuição dessas 13 profissões em cada página foi totalmente aleatória e variou de sujeito para sujeito.

As instruções dadas para os sujeitos, independentes do método escalar utilizado, requeriam que os julgamentos fossem realizados em termos do prestígio atribuído a uma dada profissão pela maioria da população. Os sujeitos que participaram em uma tarefa não foram os mesmos que participaram da outra tarefa. 0 experimento foi realizado num laboratório e os sujeitos fizeram os julgamentos individualmente.

\section{RESULTADOS E DISCUSSÃO}

Na Tabela 1 estão apresentadas as médias geométricas das estimativas de magnitudes dos prestígios de cada profissão julgados pelos 23 sujeitos (23 estimativas, 1 por sujeito, por profissão) e as médias aritméticas das estimativas de categorias expandidas dos prestígios de cada profissão julgados pelos 22 sujeitos (22 estimativas; um por sujeito por profissão). Também estão apresentadas as ordenações das posições dos julgamentos de prestígios das profissões resultantes de cada método escalar.

Pode-se observar nitidamente nesta tabela, que no método de estimação de magnitudes a profissão de Físico foi julgada como a de menor prestígio $\left(13^{\mathrm{a}}\right)$, seguida pela de Sociólogo $\left(12^{\mathrm{a}}\right)$. A profissão de Médico foi julgada como a de maior prestígio $\left(1^{\mathrm{a}}\right)$ dentre as profissões. No método de estimação de categorias expandidas a profissão de Biólogo (13a) foi julgada como a de menor prestígio, seguida pela de Assistente Social $\left(12^{\mathrm{a}}\right)$ e de Sociólogo (11 $\left.{ }^{\mathrm{a}}\right)$. A profissão de Médico foi considerada como a de maior prestígio $\left(1^{\mathrm{a}}\right)$.
A profissão de Enfermeiro ocupa a $7^{\mathrm{a}}$ posição em termos do prestígio profissional no método de estimação de magnitudes, enquanto que a mesma ocupa a $8^{a}$ posição no de estimação de categorias expandidas. Os dados obtidos neste experimento com o método de estimação de magnitudes também nos permite afirmar que o prestígio do Dentista $(E M=24,41)$ é aproximadamente duas vezes maior que a do Fonoaudiólogo ( $E M=12,27$ ) ou do Enfermeiro ( $E M=12,34$ ), ou mesmo que o prestígio do Assistente Social $(E M=10,94)$ é aproximadamente metade da do Engenheiro (EM $=22,22)$. Com dados desta natureza quaisquer outras razões entre as diferentes profissões podem ser admissiveis. De outro lado, com os dados obtidos através do método de estimação de categorias podemos afirmar que os graus de prestígios das profissões são diferentes, mas nada podemos afirmar a respeito das razões entre os graus das profissões.

Tabela 1 - Média geométrica das estimativas de magnitudes (EM), média aritmética das estimativas de categorias expandidas (ECE) e ordenação das posições (OP) de prestígios das diferentes profissões para cada método

\begin{tabular}{|c|c|c|c|c|}
\hline PROFISSÕES & EH & op & ECE & $\mathbf{O P}$ \\
\hline Químico & 10,21 & $10^{\mathrm{a}}$ & 14,19 & $9^{\mathrm{a}}$ \\
\hline Sociólogo & 9,62 & $12^{\mathrm{a}}$ & 13,36 & $11^{\mathrm{a}}$ \\
\hline Dentista & 24,41 & $2^{\mathrm{a}}$ & 33,71 & $2^{a}$ \\
\hline Engenheiro & 22,22 & $3^{\mathrm{a}}$ & 32,52 & $3^{a}$ \\
\hline Farmacêutico & 14,71 & $6^{a}$ & 18,62 & $5^{a}$ \\
\hline Biólogo & 9,95 & $11^{\mathrm{a}}$ & 12,05 & $13^{\mathrm{a}}$ \\
\hline ENFERMEIRO & 12,34 & $7^{a}$ & 15,67 & $\widehat{\diamond}^{\mathrm{a}}$ \\
\hline Médico & 29,01 & $1^{a}$ & $3 \otimes, 62$ & $1^{\mathrm{a}}$ \\
\hline Físico & 9,27 & $13^{\mathrm{a}}$ & 13,95 & $10^{\mathrm{a}}$ \\
\hline Assistente Social & 10,94 & $9^{\mathrm{a}}$ & 12,63 & $12^{\mathrm{a}}$ \\
\hline Fisioterapeuta & 14,98 & $5^{a}$ & 17,90 & $6^{3}$ \\
\hline Psicólogo & 16,68 & $4^{3}$ & 22,29 & $4^{a}$ \\
\hline Fonoaudiólogo & 12,27 & $\hat{\gamma}^{\mathrm{a}}$ & 16,76 & $7^{3}$ \\
\hline
\end{tabular}

$\mathrm{Na}$ Figura 1, as médias aritméticas das estimativas de categorias expandidas são projetadas em função das médias geométricas das estimativas de magnitudes. Essas estimativas de categorias são linearmente $\left(r^{2}=0,98\right)$ relacionadas às estimativas de magnitudes, significando que a partir do momento em que aumentamos a amplitude limitada das categorias, esse método passa a ter as mesmas características da estimação de magnitude, ou seja, o contínuo passa a ter mais uma característica que o qualifique como protético. Quando colocadas em coordenadas logarítmicas, tal como representadas na Figura 2, a relação pode ser descrita por uma função de potência com um expoente igual a $0,94\left(r^{2}=0,96\right)$. Este valor não é significativamente diferente da unidade ( $t(11)=-1,10, p=0,29$ ). Esse padrão de resultados obtido com o contínuo não métrico de prestígio profissional é muito similar com aqueles obtidos com contínuos métricos $^{(8-10)}$. 
Como nos dois experimentos anteriores, os erros padrão das médias geométricas aumentam linearmente em função das estimativas de magnitudes $\left(r^{2}=0,75\right)$ indicando que a variabilidade das estimativas é uma função linear da magnitude das estimativas. Portanto, esta relação representada na Figura 3 novamente confirma a Lei de Ekman. Ao contrário, quando os erros padrão das médias aritméticas são projetados em função das estimativas de categorias com amplitude expandida, a função, como representada na Figura 4, é inversa. Em outras palavras, quanto maior a estimativa de categoria tanto menor o erro padrão da média aritmética ou tanto menor a variabilidade. Portanto, mesmo quando aumenta-se a amplitude de categorias, mas se mantém as instruções para que os sujeitos julguem diferenças e não razões entre os graus de prestígios, a variabilidade indicada pelo erro padrão diminui com o aumento das estimativas de categorias.

Em resumo, os dados deste experimento evidenciam que é necessário atingir pelo menos um dentre dois critérios para afirmar se um contínuo não métrico tem características protéticas ou não. 0 primeiro critério seria verificar a função quase-logarítmica entre as estimativas de magnitudes e as estimativas de categorias. $O$ segundo critério seria verificar se os dados seguem a Lei de Ekman, ou seja, que a variabilidade é uma função linear do aumento das estimativas.

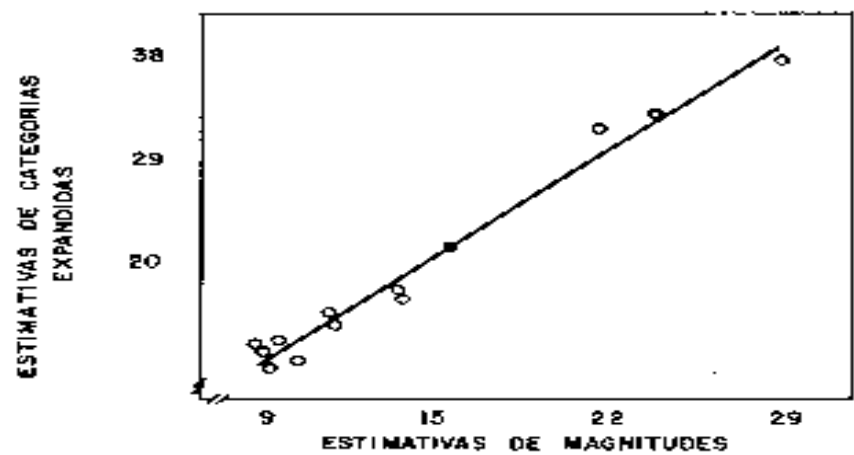

Figura 1 - Relação entre as médias aritméticas das estimativas de categorias expandidas e as médias das estimativas de magnitudes do prestígio de diferentes profissões em coordenadas lineares. As categorias variaram de 1 a 40

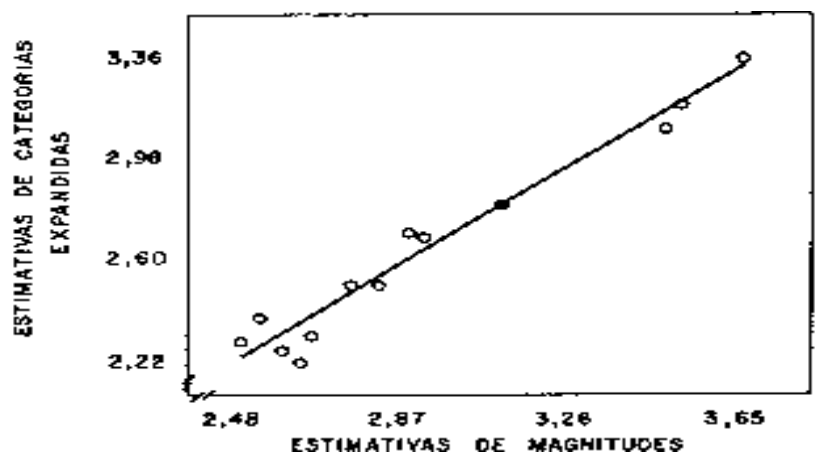

Figura 2 - Relação entre os logaritmos das médias aritméticas das estimativas de categorias expandidas e os logaritmos das estimativas de magnitudes de diferentes profissões. As categorias variaram de 1 a 40

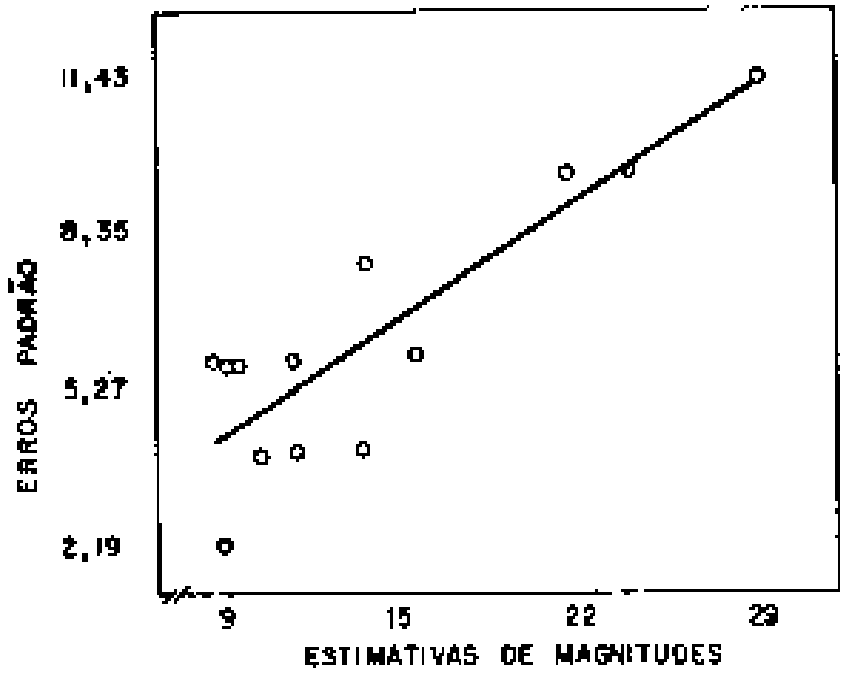

Figura 3 - Erro padrão da média geométrica em função da média geométrica das estimativas de magnitudes do prestígio de diferentes profissões

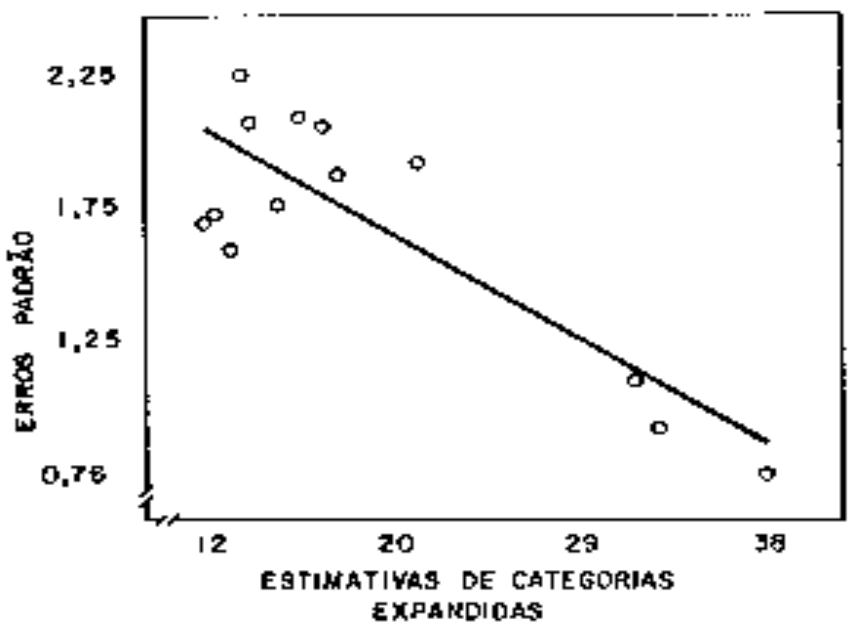

Figura 4 - Erro padrão da média aritmética das estimativas de categorias expandidas do prestígio de diferentes profissões. As categorias variaram de 1 a 40

\section{CONCLUSÕES}

Os dados do experimento realizado neste estudo permitemnos concluir que (1) a profissão de Enfermeiro ocupa a sétima ou a oitava posição em termos do prestígio das 13 profissões, enquanto a de médico ocupa a primeira posição nas escalas obtidas pelos métodos utilizados (2) as ordenações resultantes dos métodos produzem posições de prestígio altamente concordantes para as diferentes profissões (3) a relação entre as estimativas de magnitudes e as estimativas de categorias expandidas é uma função de potência com um expoente não significativamente diferente de 1,0. 


\section{REFERÊNCIAS BIBLIOGRÁFICAS}

1. Perloe SI. The relation between category-rating and magnitudeestimation judgments of occupational prestige. Am J Psychol 1963; 76:395-403.

2. Künnapas TM, Wikstroem I. Measurement of occupational preferences: A comparison of scaling methods. Percept Motor Skills 1963; 17:611-24.

3. Wegener B. Social Attitudes and Psychophysical Measurement. Hillsdale (NJ): LEA; 1982.

4. Dawson WE, Brinker RP. Validation of ratio scales of opinion by multimodality matching. Percept Psychophys 1971; 9:413-7.

5. Dawson WE, Mirando MA. Inverse scales of opinion obtained by sensory-modality matching. Percept Motor Skills 1976; 42:415-25.
6. Hardin C, Birnbaum MC. Malleability of "ratio" judgments of occupational prestige. Am J Psychol 1990; 103:1-20.

7. Faleiros Sousa FAE, Da Silva JA. Psicofísica do prestígio social: comparação entre estimação de magnitudes e de comparação aos pares. Arq Bras Psicol 1996; 48(3):69-79.

8. Guirao M. Estimaciones numéricas de brillo y luminosidad com y sin rangos fijos. In: Da Silva JA, editor. Percepcion y Psicofisica. Bogotá: Editorial ABC; 1987. p. 297-318.

9. Guirao M. A single scale based on ration and partition estimates. In: Bolanowski SJ, Gescheider GA, editors. Ratio scaling of psychological magnitude: In: Honor of the Memory of Stevens, SS Hillsdale. New Jersey: LEA; 1991. p.59-78.

10. Gibson RH, Tomko DL. The relation between magnitude and category estimates of tactile intensity. Percept Psychophys 1972; 12:135-8. 